\title{
Particle Collision Near 1 + 1-Dimensional Horava-Lifshitz Black Hole and Naked Singularity
}

\author{
M. Halilsoy and A. Ovgun \\ Physics Department, Eastern Mediterranean University, Gazimagusa, Northern Cyprus, Mersin 10, Turkey \\ Correspondence should be addressed to A. Ovgun; ali.ovgun@emu.edu.tr
}

Received 5 October 2016; Revised 29 November 2016; Accepted 27 December 2016; Published 16 January 2017

Academic Editor: Tiberiu Harko

Copyright ( 2017 M. Halilsoy and A. Ovgun. This is an open access article distributed under the Creative Commons Attribution License, which permits unrestricted use, distribution, and reproduction in any medium, provided the original work is properly cited. The publication of this article was funded by $\mathrm{SCOAP}^{3}$.

\begin{abstract}
The unbounded center-of-mass (CM) energy of oppositely moving colliding particles near horizon emerges also in $1+1$ dimensional Horava-Lifshitz gravity. This theory has imprints of renormalizable quantum gravity characteristics in accordance with the method of simple power counting. Surprisingly the result obtained is not valid for a 1-dimensional Compton-like process between an outgoing photon and an infalling massless/massive particle. It is possible to achieve unbounded CM energy due to collision between infalling photons and particles. The source of outgoing particles may be attributed to an explosive process just outside the horizon for a black hole and the naturally repulsive character for the case of a naked singularity. It is found that absence of angular momenta in 1 +1-dimension does not yield unbounded energy for collisions in the vicinity of naked singularities.
\end{abstract}

\section{Introduction}

It is known that in spacetime dimensions less than four gravity has no life of its own unless supplemented by external sources. With that addition we can have lower dimensional gravity and we can talk of black holes, wormholes, geodesics, lensing effect, and so on in analogy with the higher dimensions. One effect that attracted much interest in recent times is the process of particle collisions near the horizon of black holes due to Bañados et al. [1] which came to be known as the BSW effect. This problem arose as a result of imitating the rather expensive venture of high energy particle collisions in laboratory. From curiosity the natural question arises: is there a natural laboratory (a particle accelerator) in our cosmos that we may extract information/energy in a cheaper way? This automatically drew attention to the strong gravity regions such as near horizon of black holes. Rotating black holes host greater energy reservoir due to their angular momenta and attention naturally focused therein first $[2,3]$. In case the metric is static and diagonal, there are reasons to consider the collision process in the vicinity of a naked singularity as well.

We note from physical grounds that outgoing particles from the event horizon of a black hole cannot occur. Hawking radiation particles/photons emerge too weak to compare with infalling particles. Thus collision of two particles can only be argued if both are infalling toward the horizon of a black hole. Such a process, however, yields no BSW effect in the nonrotating metrics, which is our main interest in this study. In order to have an unbounded CM energy in a collision process both particles must be taken in the same coordinate frame and in opposite directions. This is possible in the vicinity of a naked singularity whose repulsive effect compels particles/photons to make collisions with an infalling particle/photon. From the outset we state that such a collision taking place near the naked singularity in the absence of angular momenta does not yield an unbounded $\mathrm{CM}$ energy. To extend our study to cover also collisions near black holes we assume that some unspecified process, such as disintegration decay process of some particles, yields outgoing particles photons while the partners fall into the hole. For a thorough analysis of all these problems covering the ergosphere region of a Kerr black hole, Penrose process, particle collisions, and so on one must consult [4].

In general one considers the radial geodesics and upon energy-momentum conservation in the center-of-mass (CM) frame the near horizon limit is checked whether the energy is bounded/unbounded. Our aim in this study is to consider black hole solutions in $1+1$-dimensional Horava-Lifshitz 
(HL) gravity [5] and check the BSW effect in such reduced dimensional theory. Let us remark that at the Planck scale in higher dimensions the spherical part $r^{2} d \Omega_{D-2}^{2}$ of the line element is less effective compared to the time and radial components. For this reason 1+1-dimension becomes significant at the Planck scale. For a number of reasons HL gravity is promising as a candidate for a renormalizable quantum gravity physics which has been yearned for a long time [6]. The key idea in HL- gravity is the inhomogeneous scaling properties of time and space coordinates which violate the Lorentz invariance. Arnowitt-Deser-Misner (ADM) splitting of space and time [7] constitutes its geometrical background. BSW effect in lower/higher dimensions has been worked out by many authors [8-44]. Following the similar idea we consider black hole solutions and naked singularities in $1+1$-dimension and search for the same effect in this lower dimension. It should be added that with $1+1$ dimensional HL theory the simplest nontrivial solution is the solution describing an accelerated particle in the flat space of Rindler frame. This justifies also the meaning of the vector field $\left(a_{i}\right)$ as the acceleration in the HL gravity. The role of Rindler acceleration in $3+1$-dimension as a possible source of flat rotation curves and geodesics motion has been discussed recently [45]. It is our belief that the results in lower dimensions are informative for higher dimensions and as a toy model can play the role as precursors in this regard. Even a Compton-like process can be considered at the toy level between a massless photon outgoing from a naked singularity and a particle falling into the naked singularity. The diverging $\mathrm{CM}$ energy results in the case of photon-particle collision in $1+1$-dimension under specific conditions.

Organization of the paper is as follows. In Section 2, we review in brief the $1+1-\mathrm{D}$ HL theory with a large class of black hole and naked singularity solutions. CM energy of colliding particles near horizon and naked singularity is considered in Section 3. Section 4 proceeds with applications to particular examples. The case of particle-photon collision is studied separately in Section 5. The paper ends with our conclusion in Section 6.

\section{1 + 1-D HL Black Hole/Naked Singularity}

HL formalism in $3+1-D$ makes use of the ADM splitting of time and space components as follows:

$$
d s^{2}=-N^{2} d t^{2}+g_{i j}\left(d x^{i}+N^{i} d t\right)\left(d x^{j}+N^{j} d t\right)
$$

where $N(t)$ and $N^{i}$ are the lapse and shift functions, respectively. The action of this theory is

$$
S=\frac{M_{P l}^{2}}{2} \int d^{3} x d t \sqrt{g}\left(K_{i j} K^{i j}+\lambda K^{2}+V(\phi)\right),
$$

where $K_{i j}$ is the extrinsic curvature tensor with trace $K$ and Planck mass $M_{P l} . V(\phi)$ stands for the potential function of a scalar field $\phi$, and $\lambda$ is a constant $(\lambda>1)$. Reduction from $3+1-\mathrm{D}$ to $1+1-\mathrm{D}$ results in the action [5]:

$$
S=\int d t d x\left(-\frac{1}{2} \eta N^{2} a_{1}^{2}+\alpha N^{2} \phi^{\prime 2}-V(\phi)\right)
$$

where $\eta=$ constant and $\alpha=$ constant will be chosen to be unity and $a_{1}=(\ln N)^{\prime}$. Let us comment that a "prime" denotes $d / d x$. We note that also the first term in $S$ is inherited from the geometric part of the action while the other two terms are from the scalar field source. For simplicity we have set also $M_{P l}=1$.

It has been shown in [5] that by variational principle a general class of solutions is obtained as follows:

$$
N(x)^{2}=2 C_{2}+\frac{A}{\eta} x^{2}-2 C_{1} x+\frac{B}{\eta x}+\frac{C}{3 \eta x^{2}}
$$

in which $C_{2}, A, C_{1}, B$, and $C$ are integration constants. Reference [5] must be consulted for the physical content of these constants.

The line element is

$$
d s^{2}=-N(x)^{2} d t^{2}+\frac{d x^{2}}{N(x)^{2}}
$$

with the scalar field

$$
\phi(x)=\ln \sqrt{2 C_{2}+\frac{A}{\eta} x^{2}-2 C_{1} x+\frac{B}{\eta x}+\frac{C}{3 \eta x^{2}}} .
$$

Note that the associated potential is

$$
V(\phi(x))=A+\frac{B}{x^{3}}+\frac{C}{x^{4}}
$$

and the Ricci scalar is calculated as

$$
R=-\frac{2}{\eta}\left(A+\frac{B}{x^{3}}+\frac{C}{x^{4}}\right) .
$$

There is naked singularity when $A=C_{1}=0$ and $C_{2}=B=$ $C=\eta=1$, so that there is no horizon for

$$
N(x)^{2}=2+\frac{1}{x}+\frac{1}{3 x^{2}}
$$

Another black hole solution reported by Bazeia et al. [5] is found by taking $C_{1} \neq 0, C_{2} \neq 0, B \neq 0$, and $A=C=0$.

$$
N(x)^{2}=2 C_{2}-2 C_{1} x+\frac{B}{\eta x} .
$$

This solution develops the following horizons:

$$
x_{h}^{ \pm}=\frac{C_{2}}{2 C_{1}} \pm \sqrt{\Delta}, \quad \Delta=\frac{C_{2}^{2}}{4 C_{1}^{2}}+\frac{B}{2 \eta C_{1}} .
$$

As $\Delta=0$ they degenerate; that is, $x_{h}^{+}=x_{h}^{-}$.

The Hawking temperature is given in terms of the outer $\left(x_{h}^{+}\right)$horizon as follows:

$$
T_{H}=\left.\frac{\left(N(x)^{2}\right)^{\prime}}{4 \pi}\right|_{x=x_{h}^{+}}
$$


For the special case $C_{2}=0, C_{1}=-M$, and $B=-2 M$ the horizons are independent of the mass $M$ :

$$
x_{h}^{ \pm}= \pm \frac{1}{\sqrt{\eta}} \quad(\eta>0) .
$$

The temperature is then given simply by

$$
T_{H}=\frac{M}{\pi} \text {. }
$$

This is a typical relation between the Hawking temperature and the mass of black holes in $1+1$-dimension [46].

In the case of $C_{2}=1 / 2, B=-2 M, \eta=1$, and $A=C=$ $C_{1}=0$ it gives a Schwarzschild-like solution;

$$
N(x)^{2}=1-\frac{2 M}{x} .
$$

On the other hand, the choice of the parameters, for $C_{2}=$ $1 / 2, B=-2 M, C=3 Q^{2}, \eta=1$, and $A=C_{1}=0$, gives a Reissner-Nordstrom-like solution.

$$
N(x)^{2}=1-\frac{2 M}{x}+\frac{Q^{2}}{x^{2}} .
$$

As in the general relativity we can make particular choice of the parameters so that we end up with a naked singularity instead of a black hole. The choice $Q^{2}>M^{2}$ in (16), for instance, transforms the HL- black hole into a naked singularity at $x=0$. Similarly $M<0$ turns (15) into a naked singular metric at $x=0$.

\section{CM Energy of Particle Collision near the Horizon of the 1+ 1-D HL Black Hole}

Here we will derive the equations of motion of an uncharged massive test particle by using the method of geodesic Lagrangian. Such equations can be derived from the Lagrangian equation,

$$
\mathscr{L}=\frac{1}{2}\left[-N(x)^{2}\left(\frac{d t}{d \tau}\right)^{2}+\frac{1}{N(x)^{2}}\left(\frac{d x}{d \tau}\right)^{2}\right],
$$

in which $\tau$ is the proper time for time-like geodesics (or massive particles). The canonical momenta are

$$
\begin{aligned}
& p_{t}=\frac{d \mathscr{L}}{d \dot{t}}=-N(x)^{2} \dot{t} \\
& p_{x}=\frac{d \mathscr{L}}{d \dot{x}}=\frac{\dot{x}}{N(x)^{2}}
\end{aligned}
$$

The $1+1$-D HL black hole has only one killing vector $\partial_{t}$. The associated conserved quantity will be labeled by $E$. From (18), $E$ is related to $N(x)^{2}$ as

$$
-N(x)^{2} \dot{t}=-E \text {. }
$$

Hence,

$$
\dot{t}=\frac{E}{N(x)^{2}}
$$

The two velocities of the particles are given by $u^{\mu}=$ $d x^{\mu} / d \tau$. We have already obtained $u^{t}$ in the above derivation. To find $u^{x}=\dot{x}$, the normalization condition for time-like particles, $u^{\mu} u_{\mu}=-1[1,47]$, can be used as

$$
g_{t t}\left(u^{t}\right)^{2}+g_{x x}\left(u^{x}\right)^{2}=-1 .
$$

By substituting $u^{t}$ to (22), one obtains $u^{x}$ as

$$
\left(u^{x}\right)^{2}=E^{2}-N(x)^{2}
$$

for which an effective potential $V_{\text {eff }}$ can be defined by

$$
\left(u^{x}\right)^{2}+V_{\text {eff }}=E^{2} \text {. }
$$

Now, the two velocities can be written as

$$
\begin{aligned}
& u^{t}=\dot{t}=\frac{E}{N(x)^{2}}, \\
& u^{x}=\dot{x}=\sqrt{E^{2}-N(x)^{2}} .
\end{aligned}
$$

We proceed now to present the CM energy of two particles with two velocities $u_{1}^{\mu}$ and $u_{2}^{\mu}$. We will assume that both have rest mass $m_{0}=1$. The CM energy is given by

$$
E_{\mathrm{cm}}=\sqrt{2} \sqrt{\left(1-g_{\mu \nu} u_{1}^{\mu} u_{2}^{\nu}\right)} \text {. }
$$

So

$$
\frac{E_{\mathrm{cm}}^{2}}{2}=1+\frac{E_{1} E_{2}}{N(x)^{2}}-\frac{\kappa \sqrt{E_{1}^{2}-N(x)^{2}} \sqrt{E_{2}^{2}-N(x)^{2}}}{N(x)^{2}},
$$

where $\kappa= \pm 1$ corresponds to particles moving in the same/opposite direction with respect to each other. We wish to stress that our concern is for the case $\kappa= \pm 1$ since no physical particle is ejected from the black hole. Note that $E_{1}$ and $E_{2}$ are the energy constants corresponding to each particle. In case the second term under the square root is too small than the first one,

$$
\sqrt{E^{2}-N(x)^{2}} \approx\left(E-\frac{N(x)^{2}}{2 E^{2}}+\cdots\right)
$$

so that the higher order terms can be neglected and CM energy of two particles can be written as [23]

$$
\frac{E_{\mathrm{cm}}^{2}}{2} \approx 1+(1-\kappa) \frac{E_{1} E_{2}}{N(x)^{2}}+\frac{\kappa}{2}\left(\frac{E_{2}}{E_{1}}+\frac{E_{1}}{E_{2}}\right) .
$$

The case with $\kappa=+1$ is obvious, in which the CM energy becomes

$$
\frac{E_{\mathrm{cm}}^{2}}{2} \approx 1+\frac{\left(E_{2}^{2}+E_{1}^{2}\right)}{2 E_{1} E_{2}}
$$

where the CM energy is independent of metric function, and it gives always a finite energy. On the other hand $\kappa=-1$ gives

$$
\frac{E_{\mathrm{cm}}^{2}}{2} \approx 1+\frac{2 E_{1} E_{2}}{N(x)^{2}}-\frac{\left(E_{2}^{2}+E_{1}^{2}\right)}{2 E_{1} E_{2}}
$$

in which it gives unbounded CM energy near the horizon of the HL black holes provided an outgoing particle mechanism from the horizon is established. Otherwise the yield of two ingoing particles collision remains finite. 


\section{Some Examples}

4.1. Schwarzschild-Like Solution. In the case of $C_{2}=1 / 2, B=$ $-2 M, \eta=1$, and $A=C=C_{1}=0$ it gives Schwarzschild-like solution, where

$$
\begin{aligned}
V(\phi(x)) & =-\frac{2 M}{x^{3}} \\
N(x)^{2} & =1-\frac{2 M}{x} .
\end{aligned}
$$

For the CM energy on the horizon, we have to compute the limiting value of (27) as $x \rightarrow x_{h}=2 M$, where the horizon of the black hole is. Setting $\kappa=-1$ as it is, the CM energy near the event horizon for $1+1 \mathrm{D}$ Schwarzschild $\mathrm{BH}$ is

$$
E_{\mathrm{cm}}^{2}\left(x \longrightarrow x_{h}\right)=\infty .
$$

This result for 4-D Schwarzschild Black hole is already calculated by Baushev [24]. Hence, the condition of $\kappa=-1$, when the location of particle 1 approaches the horizon and on the other hand the particle 2 runs outward from the horizon due to some unspecified physical process, yet yields $E_{\mathrm{cm}}^{2} \rightarrow$ $\infty$ so there is BSW effect for $1+1$ Schwarzschild-like solution when the condition $\kappa=-1$ is satisfied.

4.2. Reissner-Nordstrom-Like Solution. On the other hand, the choice of the parameters, for $C_{2}=1 / 2, B=-2 M$, $C=3 Q^{2}, \eta=1$, and $A=C_{1}=0$ gives the ReissnerNordstrom-like solution.

$$
\begin{gathered}
N(x)^{2}=1-\frac{2 M}{x}+\frac{Q^{2}}{x^{2}}, \\
V(\phi(x))=-\frac{2 M}{x^{3}}+\frac{3 Q^{2}}{x^{4}}
\end{gathered}
$$

So the CM energy is calculated by using the limiting value of (31)

$$
E_{\mathrm{cm}}^{2}\left(x \longrightarrow x_{h=M+\sqrt{\left(M^{2}-Q^{2}\right)}}\right)=\infty .
$$

So there is a BSW effect.

4.3. The Extremal Case of the Reissner-Nordstrom-Like Black Hole. For the extremal case we have with $M=Q$, from (34),

$$
N(x)^{2}=\left(1-\frac{M}{x}\right)^{2}
$$

so that it also gives the same answer from (31) as

$$
E_{\mathrm{cm}}^{2}\left(x \longrightarrow x_{h}\right)=\infty \text {. }
$$

4.4. Specific New Black Hole Case. The new 3-parameter black hole solution given by Bazeia et al. [5] is chosen as

$$
N(x)^{2}=2 C_{2}-2 C_{1} x+\frac{B}{\eta x}
$$

with the potential

$$
V(\phi(x))=\frac{B}{x^{3}}
$$

For the special case $C_{2}=0, C_{1}=-M$, and $B=-2 M$ we have

$$
N(x)^{2}=2 M x-\frac{2 M}{\eta x}
$$

with suitable potential which is

$$
V(\phi(x))=-\frac{2 M}{x^{3}} .
$$

The CM energy of two colliding particles is calculated by taking the limiting values of (31)

$$
E_{\mathrm{cm}}^{2}\left(x \longrightarrow x_{h}\right)=\infty
$$

Hence the BSW effect arises here as well.

4.5. Near Horizon Coordinates. We have explored the region near the horizon by replacing $r$ by a coordinate $\rho$. The proper distance from the horizon $\rho$ [48] is given as follows:

$$
\rho=\int \sqrt{g_{x x}\left(x^{\prime}\right)} d x^{\prime}=\int_{x_{h}}^{x} \frac{1}{N\left(x^{\prime}\right)} d x^{\prime} .
$$

is

The first example is the Schwarzschild-like solution which

$$
N(x)^{2}=1-\frac{2 M}{x}
$$

so that proper distance is calculated as

$$
\begin{aligned}
\rho & =\int_{x_{h}}^{x}\left(1-\frac{2 M}{x}\right)^{-1 / 2} d x^{\prime} \\
& =\sqrt{x(x-2 M)}+2 M G \sinh ^{-1}\left(\sqrt{\frac{x}{2 M}-1}\right) .
\end{aligned}
$$

The new metric is

$$
d s^{2}=-\left(1-\frac{2 M}{x(\widetilde{\rho})}\right) d t^{2}+d \widetilde{\rho}^{2},
$$

where $\widetilde{\rho} \simeq 2 \sqrt{2 M(x-2 M)}$ so that it gives approximately

$$
d s^{2} \simeq-\frac{\rho^{2}}{(4 M)^{2}} d t^{2}+d \rho^{2}
$$

which is once more the Rindler-type line element. Let us note that this Rindler-type line element is valid within the near horizon limit approximation. For practical purposes there are advantages in adapting such an approximation which conforms with the equivalence principle [48]. The CM energy of two colliding particles is given by

$$
\begin{aligned}
& \frac{E_{\mathrm{cm}}^{2}}{2 m_{0}^{2}}=1 \\
& +\frac{(4 M)^{2}\left(E_{1} E_{2}-\kappa \sqrt{E_{1}^{2}-\rho^{4} /(4 M)^{4}} \sqrt{E_{2}^{2}-\rho^{4} /(4 M)^{4}}\right)}{\rho^{2}}
\end{aligned}
$$




\section{Particle Collision near the Naked Singularity}

There is a naked singularity for our $1+1$-D HL model at the location of $x=0$, with $Q^{2}>M^{2}$ in (16). In addition $M<0$ turns (15) into a naked singular metric at $x=0$. There is also naked singularity when we choose metric function as follows:

$$
N(x)^{2}=2+\frac{1}{x}+\frac{1}{3 x^{2}}=\frac{6 x^{2}+3 x+1}{3 x^{2}} .
$$

As it is given in (27), CM energy of the collision of two particles generally is (for $N(x) \rightarrow \infty$ ).

$$
\frac{E_{\mathrm{cm}}^{2}}{2} \approx 1-\kappa+\frac{1}{2 N(x)^{2}}\left[2 E_{1} E_{2}+\kappa\left(E_{1}^{2}+E_{2}^{2}\right)\right] \text {. }
$$

For the case $\kappa= \pm 1$, when $x$ goes to zero, the CM energy remains finite for radially moving particles.

$$
\left.\frac{E_{\mathrm{c.m} .}^{2}}{2}\right|_{x=0} \longrightarrow 1-\kappa .
$$

This suggests that although one of the particle is boosted by the naked singularity, there is not any unlimited collisional energy near such singularity. Note that Compton-like processes were considered first in [4], where rotational effect of Kerr black hole played a significant role. Our case here is entirely free of rotational effects.

\section{Photon versus an Infalling Particle}

A massless photon can naturally scatter an infalling particle or vice versa. This phenomenon is analogous to a Compton scattering taking place in $1+1$-dimension. Null-geodesics for a photon can be described simply by

$$
\begin{aligned}
& \frac{d t}{d \lambda}=\frac{E_{1}}{N^{2}} \\
& \frac{d x}{d \lambda}= \pm \sqrt{E_{1}^{2}-N^{2}}
\end{aligned}
$$

where $\lambda$ is an affine parameter and $E_{1}$ stands for the photon energy. Defining $E_{1}=\hbar \omega_{0}$, where $\omega_{0}$ is the frequency (with the choice $\hbar=1$ ) we can parametrize energy of the photon by $\omega_{0}$ alone. The CM energy of a photon and the infalling particle can be taken now as

$$
E_{\mathrm{cm}}^{2}=-\left(p^{\mu}+k^{\mu}\right)^{2}
$$

in which $p^{\mu}=m u^{\mu}$ and $k^{\mu}$ refer to the particle and photon, 2 momenta, respectively. This amounts to

$$
E_{\mathrm{cm}}^{2}=m^{2}-2 m g_{\mu \nu} u^{\mu} k^{\nu},
$$

where we have for the particle

$$
p^{\mu}=m\left(\frac{E_{2}}{N^{2}}, \sqrt{E_{2}^{2}-N^{2}}\right)
$$

and for the photon

$$
k^{\mu}=\left(\frac{E_{1}}{N^{2}},-E_{1}\right)
$$

One obtains

$$
E_{\mathrm{cm}}^{2}=m^{2}+\frac{2 m E_{1}}{N^{2}}\left(E_{2}+\kappa \sqrt{E_{2}^{2}-N^{2}}\right) .
$$

In the near horizon limit this reduces to

$$
E_{\mathrm{cm}}^{2}=m^{2}+\frac{2 m E_{1}}{N^{2}}\left(E_{2}+\kappa E_{2}-\frac{N^{2}}{2 E_{2}}\right) .
$$

Note that for $\kappa=-1$ we have $E_{\mathrm{cm}}^{2}$ given by

$$
E_{\mathrm{cm}}^{2}=m^{2}\left(1-\frac{E_{1}}{m E_{2}}\right)
$$

which is finite between the collision of a photon and an infalling particle and therefore is not of interest. As a matter of fact the occurrence of outgoing photon from the event horizon cannot be justified unless an explosive/decay process is assumed to take place. As a result for $\kappa=+1$ from (59) we obtain an unbounded $E_{\mathrm{cm}}^{2}$ between the collision of infalling photon and particle. Let us add that "inverse" Compton process in the ergosphere of Kerr black hole was considered in [4] where the photon's energy showed increment due to rotational and curvature effects. The energy, however, attained an upper bound which was finite. Our result obtained here being entirely radial on the other hand can hardly be compared with those of [4].

\section{Conclusion}

Our aim was to investigate whether the BSW type effect which arises in higher dimensional black holes applies also to the $1+1-\mathrm{D}$ naked singularity/black hole. The theory we adapted is not general relativity but instead the recently popular HL gravity. We employed the class of 5-parameter black hole/naked singularity solutions found recently [5]. The class has particular limits of flat Rindler, Schwarzschild, and Reissner-Nordstrom-like solutions. For each case we have calculated the center-of-mass (CM) energy of the particles and shown that the energy can grow unbounded for some cases. In other words the strong gravity near the event horizon affects the collision process with unlimited source to turn it into a natural accelerator. The model we use applies also to the case of a photon/particle collision with different characteristics. It is observed that the CM energy of the infalling particles from the rest at infinity will remain finite in the CM frame at the event horizon of a black hole. Contrariwise, unlimited CM energy will be attained between the collision of the outgoing particles from the event horizon region and infalling particles. It is also possible to achieve the infinite energy between an infalling photon and an infalling massive particle. However, we found finite CM energy between an outgoing photon and infalling particle. Finally, we must admit that absence of rotational effects in $1+1-\mathrm{D}$ restricts the problem to the level of a toy model in which particles move on pure radial geodesics yielding finite $\mathrm{CM}$ energy in the vicinity of a naked singularity. 


\section{Disclosure}

This work was presented as a poster at Karl Schwarzschild Meeting 20-24 July 2015 Frankfurt Institute for Advanced Studies.

\section{Competing Interests}

The authors declare that they have no competing interests.

\section{References}

[1] M. Bañados, J. Silk, and S. M. West, "Kerr black holes as particle accelerators to arbitrarily high energy," Physical Review Letters, vol. 103, no. 11, Article ID 111102, 2009.

[2] T. Jacobson and J. P. Sotiriou, "Spinning black holes as particle accelerators," Physical Review Letters, vol. 104, no. 2, Article ID 021101, 3 pages, 2010.

[3] K. Lake, "Particle accelerators inside spinning black holes," Physical Review Letters, vol. 104, Article ID 211102, 2010.

[4] T. Piran and J. Shaham, "Upper bounds on collisional penrose processes near rotating black-hole horizons," Physical Review D, vol. 16, no. 6, pp. 1615-1635, 1977.

[5] D. Bazeia, F. A. Brito, and F. G. Costa, "Two-dimensional Horava-Lifshitz black hole solutions," Physical Review D. Particles, Fields, Gravitation, and Cosmology, vol. 91, no. 4, Article ID 044026, 2015.

[6] P. Horava, "Quantum gravity at a Lifshitz point," Physical Review $D$, vol. 79, no. 8, Article ID 084008, 2009.

[7] R. Arnowitt, S. Deser, and C. W. Misner, "Republication of: the dynamics of general relativity," General Relativity and Gravitation, vol. 40, no. 9, pp. 1997-2027, 2008.

[8] E. Berti, V. Cardoso, L. Gualtieri, F. Pretorius, and U. Sperhake, "Comment on "kerr black holes as particle accelerators to arbitrarily high energy", Physical Review Letters, vol. 103, no. 23, Article ID 239001, 2009.

[9] M. Bañados, B. Hassanain, J. Silk, and S. M. West, "Emergent flux from particle collisions near a Kerr black hole," Physical Review D, vol. 83, no. 2, Article ID 023004, 2011.

[10] T. Jacobson and T. P. Sotiriou, "Spinning black holes as particle accelerators," Physical Review Letters, vol. 104, no. 2, Article ID 021101, 2010.

[11] O. B. Zaslavskii, "Acceleration of particles by nonrotating charged black holes," JETP Letters, vol. 92, no. 9, pp. 571-574, 2011.

[12] S. W. Wei, Y. X. Liu, H. T. Li, and F. W. Chen, "Particle collisions on stringy black hole background," Journal of High Energy Physics, vol. 2010, no. 12, article 066, 2010.

[13] O. B. Zaslavskii, "Energy extraction from extremal charged black holes due to the Banados-Silk-West effect," Physical Review D, vol. 86, Article ID 124039, 2012.

[14] H. Saadat, "The centre-of-mass energy of two colliding particles in STU black holes," Canadian Journal of Physics, vol. 92, no. 12, pp. 1562-1564, 2014.

[15] N. Tsukamoto and C. Bambi, "High energy collision of two particles in wormhole spacetimes," Physical Review D. Particles, Fields, Gravitation, and Cosmology, vol. 91, no. 8, Article ID 084013, 2015.

[16] S. G. Ghosh, P. Sheoran, and M. Amir, "Rotating Ayón-BeatoGarcía black hole as a particle accelerator," Physical Review D, vol. 90, no. 10, Article ID 103006, 2014.
[17] A. Galajinsky, "Particle collisions on near horizon extremal Kerr background," Physical Review D, vol. 88, no. 2, Article ID 027505, 2013.

[18] V. P. Frolov, "Weakly magnetized black holes as particle accelerators," Physical Review D, vol. 85, no. 2, Article ID 024020, 2012.

[19] A. M. Al Zahrani, V. P. Frolov, and A. A. Shoom, "Critical escape velocity for a charged particle moving around a weakly magnetized Schwarzschild black hole," Physical Review D, vol. 87, no. 8, Article ID 084043, 2013.

[20] J. Sadeghi and B. Pourhassan, "Particle acceleration in HoravaLifshitz black holes," The European Physical Journal C, vol. 72, no. 4, article 1984, 2012.

[21] J. Sadeghi, B. Pourhassan, and H. Farahani, "Rotating charged hairy black hole in $(2+1)$ dimensions and particle acceleration," Communications in Theoretical Physics, vol. 62, no. 3, pp. 358362, 2014.

[22] C. Liu, S. Chen, C. Ding, and J. Jing, "Particle acceleration on the background of the Kerr-Taub-NUT spacetime," Physics Letters. B, vol. 701, no. 3, pp. 285-290, 2011.

[23] M. Patil and P. S. Joshi, "Ultrahigh energy particle collisions in a regular spacetime without black holes or naked singularities," Physical Review D, vol. 86, no. 4, Article ID 044040, 2012.

[24] A. N. Baushev, "Dark matter annihilation in the gravitational field of a black hole," International Journal of Modern Physics D, vol. 18, no. 8, pp. 1195-1203, 2009.

[25] M. Patil and P. S. Joshi, "Particle acceleration by MajumdarPapapetrou di-hole," General Relativity and Gravitation, vol. 46, no. 10, 2014.

[26] J. D. Schnittman, "Revised upper limit to energy extraction from a kerr black hole," Physical Review Letters, vol. 113, no. 26, Article ID 261102, 2014.

[27] M. Patil and P. S. Joshi, "Naked singularities as particle accelerators," Physical Review D, vol. 82, no. 10, Article ID 104049, 2010.

[28] M. Patil, P. S. Joshi, and D. Malafarina, "Naked singularities as particle accelerators. II," Physical Review D, vol. 83, no. 6, Article ID 064007, 2011.

[29] A. Grib and Y. Pavlov, "On particle collisions in the gravitational field of the Kerr black hole," Astroparticle Physics, vol. 34, no. 7, pp. 581-586, 2011.

[30] M. Sharif and N. Haider, "Study of center of mass energy by particles collision in some black holes," Astrophysics and Space Science, vol. 346, no. 1, pp. 111-117, 2013.

[31] I. Hussain, M. Jamil, and B. Majeed, "A slowly rotating black hole in horava-lifshitz gravity and a $3+1$ dimensional topological black hole: motion of particles and BSW mechanism," International Journal of Theoretical Physics, vol. 54, no. 5, pp. 1567-1577, 2015.

[32] S. Hussain, I. Hussain, and M. Jamil, "Dynamics of a charged particle around a slowly rotating Kerr black hole immersed in magnetic field," The European Physical Journal C, vol. 74, no. 12, 2014.

[33] M. Amir and S. G. Ghosh, "Rotating Hayward's regular black hole as particle accelerator," Journal of High Energy Physics, vol. 2015, no. 7, article 015, 2015.

[34] B. Pourhassan and U. Debnath, "Particle acceleration in rotating modified hayward and bardeen black holes," https://arxiv.org/ abs/1506.03443.

[35] A. A. Grib and Y. V. Pavlov, "Are black holes totally black?" Gravitation and Cosmology, vol. 21, no. 1, pp. 13-18, 2015. 
[36] A. A. Grib and Y. V. Pavlov, "High energy physics in the vicinity of rotating black holes," Theoretical and Mathematical Physics, vol. 185, no. 1, pp. 1425-1432, 2015.

[37] C. Ding, C. Liu, and Q. Quo, "Spacetime noncommutative effect on black hole as particle accelerators," International Journal of Modern Physics D, vol. 22, no. 04, Article ID 1350013, 2013.

[38] J. Yang, Y.-L. Li, Y. Li, S.-W. Wei, and Y.-X. Liu, "Particle collisions in the lower dimensional rotating black hole spacetime with the cosmological constant," Advances in High Energy Physics, vol. 2014, Article ID 204016, 7 pages, 2014.

[39] H. Nemoto, U. Miyamoto, T. Harada, and T. Kokubu, "Escape of superheavy and highly energetic particles produced by particle collisions near maximally charged black holes," Physical Review D, vol. 87, no. 12, Article ID 127502, 2013.

[40] C. Zhong and S. Gao, "Particle collisions near the cosmological horizon of a Reissner-Nordström-de Sitter black hole," JETP Letters, vol. 94, no. 8, pp. 589-592, 2011.

[41] C. Liu, S. Chen, and J. Jing, "Collision of two general geodesic particles around a kerr-newman black hole," Chinese Physics Letters, vol. 30, no. 10, Article ID 100401, 2013.

[42] Y. Zhu, S. Wu, Y. Liu, and Y. Jiang, "General stationary charged black holes as charged particle accelerators," Physical Review D, vol. 84, no. 4, Article ID 043006, 2011.

[43] U. Miyamoto, H. Nemoto, and M. Shimano, "Particle creation by naked singularities in higher dimensions," Physical Review D, vol. 83, no. 8, Article ID 084054, 2011.

[44] Y. Li, J. Yang, Y.-L. Li, S.-W. Wei, and Y.-X. Liu, "Particle acceleration in Kerr-(anti-)de Sitter black hole backgrounds," Classical and Quantum Gravity, vol. 28, no. 22, Article ID 225006, 2011.

[45] M. Halilsoy, O. Gurtug, and S. H. Mazharimousavi, "Rindler modified Schwarzschild geodesics," General Relativity and Gravitation, vol. 45, no. 11, pp. 2363-2381, 2013.

[46] S. W. Hawking, "Black holes and thermodynamics," Physical Review D, vol. 13, no. 2, pp. 191-197, 1976.

[47] C. W. Misner, K. S. Thorne, and J. A. Wheeler, Gravitation, W.H. Freeman \& Co., San Francisco, Calif, USA, 1972.

[48] L. Susskind and J. Lindesay, An Introduction to Black Holes, Information and the String Theory Revolution: The Holographic Universe, World Scientific, Hackensack, NJ, USA, 2005. 

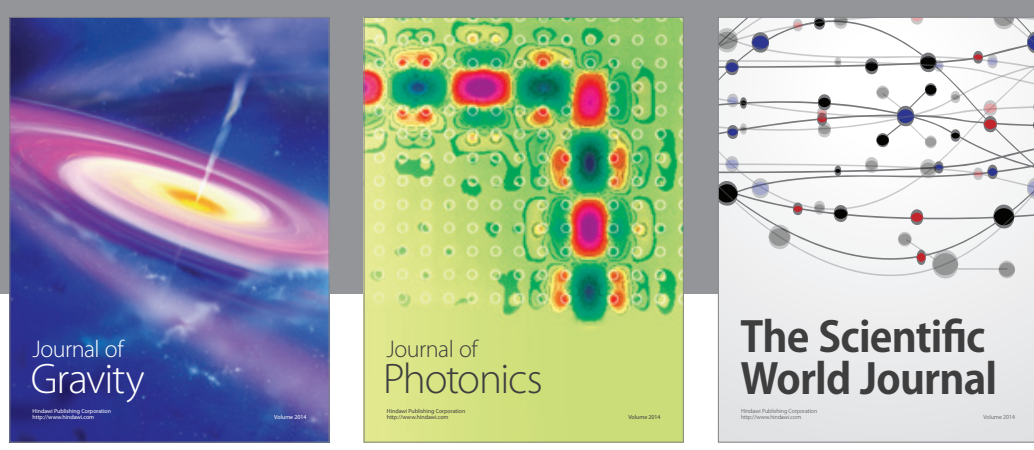

The Scientific World Journal
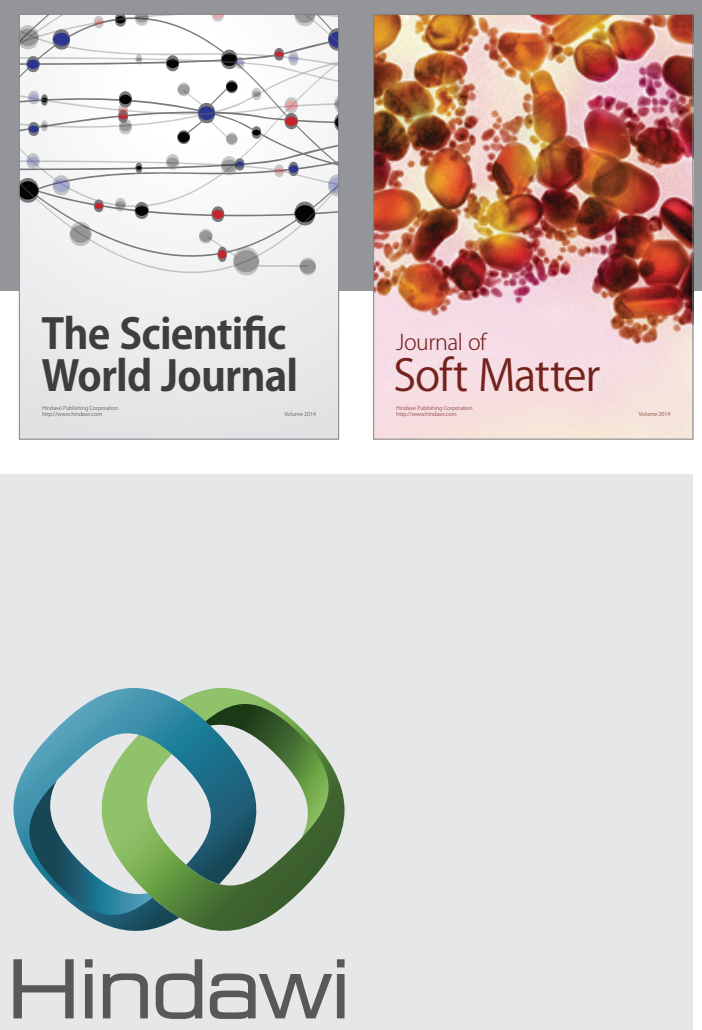

Submit your manuscripts at

https://www.hindawi.com
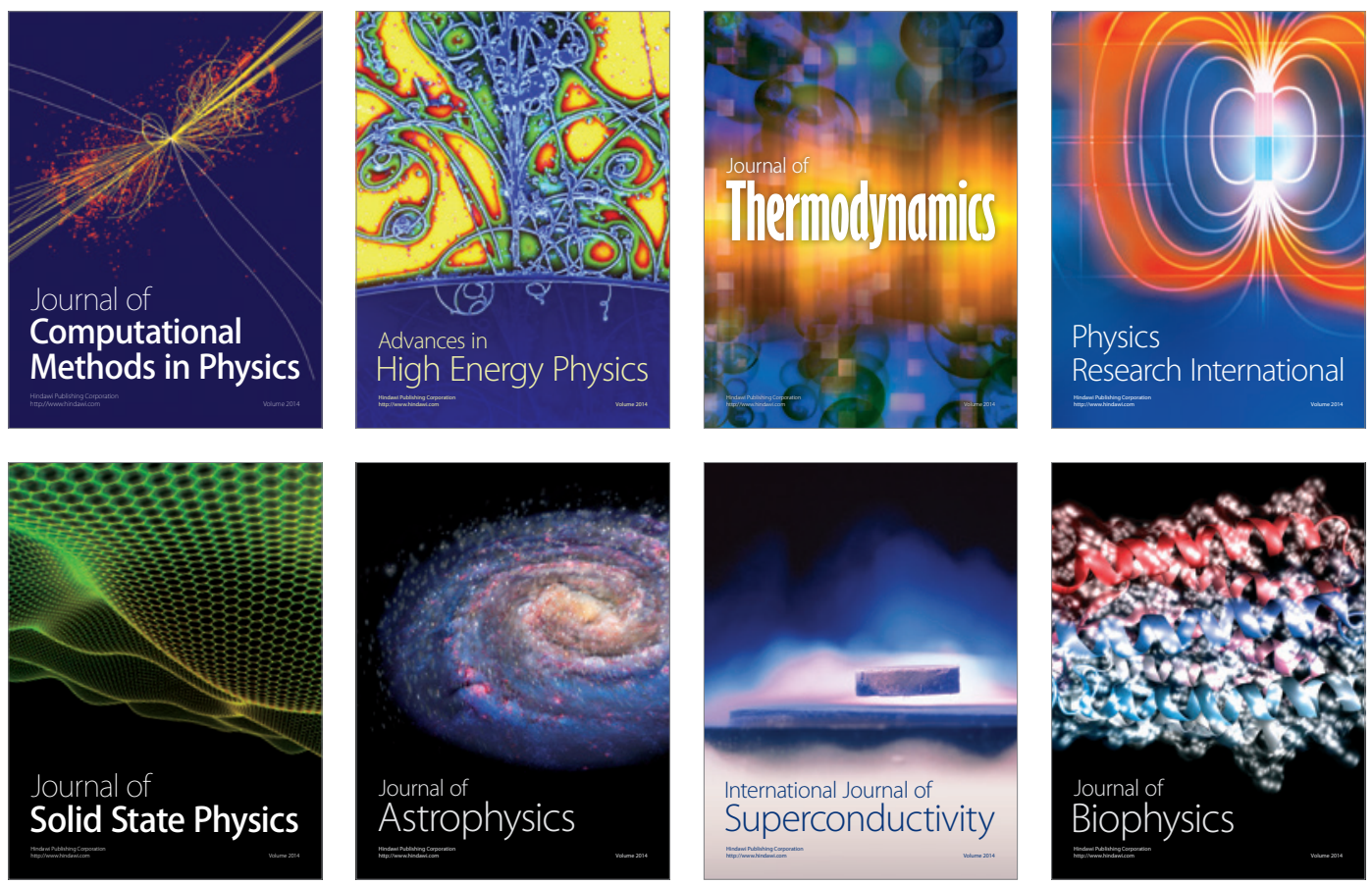
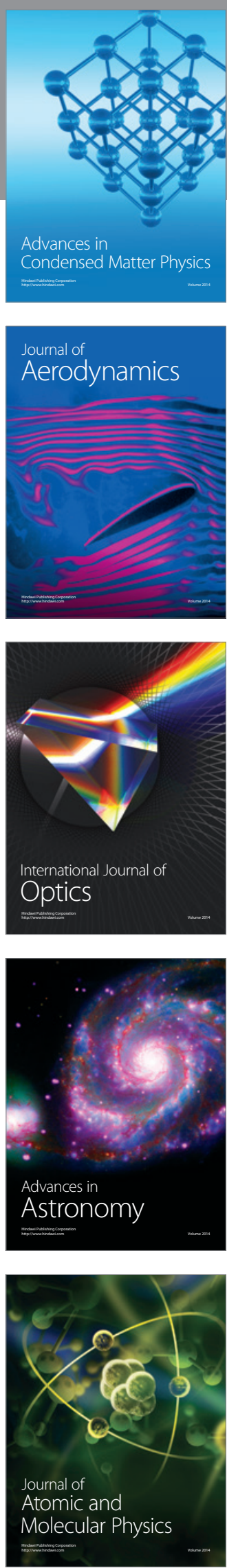